\title{
Preferential MBE Growth and Characterization of SiGe Nanoislands on Depth-Selective Si Pits Etched by $\mathrm{Ar}^{+}$Plasma
}

\author{
Isa Şeker,* Ali Karatutlu,* and Sümeyra İstengir
}

In this study, the size selective deposition of SiGe nanoislands is demonstrated to be possible only in Si nanopits using a molecular beam epitaxy (MBE) system. The depth of the etched Si substrate prepared by $\mathrm{Ar}^{+}$plasma etching just before the deposition seems to be playing a role in the selectivity of keeping the SiGe nanoislands only inside the nanopits. We observed that, when the thickness of the deposited SiGe layer is around the mean pit depth, which is $4 \mathrm{~nm}$ in this case, Ge nucleation takes place selectively on the preetched pits. Relatively larger deposition thickness (e.g., $40 \mathrm{~nm}$ ) is demonstrated to suppress the preferential growth of the Ge nanocrystals (NCs)/Si NCs which are in return observed all along the surface of the Si substrate. On the other hand, surface migration is considered to play a role in very small depth (relatively more shallow pits) and yielding the unfilled Si nanopits (ca. $1.5 \mathrm{~nm}$ ) whereas Ge NCs selectively nucleate only within those having larger depths $(c a .3 \mathrm{~nm})$. Such site-specific 3D controlled growth of nanoislands is shown for the deposition of different semiconductor nanocrystals on top of another for formation of nanodevices fabricated in a single nanopit.

years, Ge together with $\mathrm{Si}$ seems to offer new insights. Ge has a low band gap $(0.67 \mathrm{eV})$ and relatively high carrier mobility $\left(\mu_{\mathrm{e}}=1900 \mathrm{~cm}^{2} \mathrm{~V}^{-1} \mathrm{~s}^{-1}\right.$ and $\mu_{\mathrm{h}}=3600$ $\left.\mathrm{cm}^{2} \mathrm{~V}^{-1} \mathrm{~s}^{-1}\right)$. Due to these properties, an opportunity arises for operation of electronic devices faster at lower applied voltage compared to its chemical analogue Si. Si and $\mathrm{Ge}$ are not only chemical analogues but these two elements also demonstrate good partnership in strain engineering using SiGe with higher carrier mobilities $\left(>10.000 \mathrm{~cm}^{2} \mathrm{~V}^{-1} \mathrm{~s}^{-1}\right)$ which can even lead to the direct band semiconductors upon 1.4 biaxial tensile strain..$^{[1]}$ Recently, electrically pumped lasing ${ }^{[2,3]}$ and high performance photodetector ${ }^{[4]}$ using $\mathrm{Ge}$ on $\mathrm{Si}$ were reported. SiGe thin film layers can be grown using numerous deposition methods such as electron beam deposition, ${ }^{[5]}$ chemical vapor deposition, ${ }^{[6]}$ and RF magnetron sputtering. ${ }^{[7]}$ However,

Miniaturization and new approaches in the design of IC elements determine the current and future of the optoelectronic field where Si appears to be dominant. Nevertheless, in recent

Dr. i. Şeker

Fatih University

Bionanotechnology Research and Development Center (BINATAM)

Buyukcekmece, 34500 Istanbul, Turkey

E-mail: isaseker05@gmail.com

Dr. A. Karatutlu

Materials Science and Nanotechnology Department

UNAM-National Nanotechnology Research Center

Bilkent University

06800 Ankara, Turkey

E-mail: ali@unam.bilkent.edu.tr

Dr. A. Karatutlu

The Institute of Materials Science and Nanotechnology

Bilkent University

06800 Ankara, Turkey

S. İstengir

Department of Physics

Yıldız Technical University

Davutpaşa, 34210 Istanbul, Turkey

The ORCID identification number(s) for the author(s) of this article can be found under https://doi.org/10.1002/pssr.201700424.

DOI: 10.1002/pssr.201700424
MBE method enables the fabrication of higher crystalline quality layers due to the purity of its ultra high vacuum medium and presents the utilization of more controlled in situ parameters before and during the deposition process. The site-selected deposition of the $\mathrm{SiGe}$ nanoislands on a pre-patterned $\mathrm{Si}$ substrate was previously shown using the MBE system despite the fact that the Si pits were obtained ex situ. First, Poydenot et al. ${ }^{[8]}$ described that Ge NCs can be deposited regularly inside the Si nanopits although the preferential growth mechanism was not fully clear. Zong et al. ${ }^{[9]}$ demonstrated that the "quasiperiodically" ordered of SiGe islands can be obtained regardless of the geometry of the Si pit. Dais et al. ${ }^{[10-12]}$ reported that shape and size of nanoislands can change according to the change of the pit diameter obtained using extreme ultraviolet interference lithography (EUV-IL) in the Swiss light source. We would like to emphasize on that the template formations in the abovementioned studies were conducted ex situ and require either one of pre-treatments such as mechanical etching, the high energetic beam exposure and/or the use of $\mathrm{HF}$ acid before the insertion to the MBE system to remove the surface oxides. Although there exist advanced multi-chamber vacuum systems with particular fabrication or characterization units, to our knowledge, in situ fabrication of ordered arrays of nanoholes or another nanopatterned structure has not been reported so far by these systems. Nano patterning of such architectures require the 
utilization of lithographic techniques such as electron beam, optical, UV i.e., lithography including successive coating, lift-off, developing processes, etc. ${ }^{[13,14]}$ Due to the UHV purity nature of these deposition systems, involvement of such wet chemistry or existence of their chemical remnants or residues cannot be tolerated. Moreover, there is no study on selectively growing SiGe nanoislands dependent on the depth of the Si pits using an MBE system. In this study, we show that $\mathrm{Ge} / \mathrm{Si} \mathrm{NCs}$ can be deposited selectively inside a pre-etched $\mathrm{Si}$ nanopit formed by $\mathrm{Ar}^{+}$plasma etching available in the MBE.

Experimental Section: The experimental part of the formation of the selective deposition of nanoislands include the subsections of substrate and instrumentation (Figure S1, Supporting Information for the MBE system), substrate cleaning, chamber preparation, dry etching and reflection high energy electron diffraction (RHEED) analysis, SiGe growth and annealing. The details of the subsections can be found in the Supporting Information.

Dry Etching: In situ dry etching with RF argon plasma was performed at two energy values of 40 and $400 \mathrm{eV}$ for $30 \mathrm{~min}$. Plasma is formed when mass flow control (MFC) system is arranged to $10 \mathrm{sccm}$, an appropriate flow of high purity Ar. The AFM images in Figure S2(b) and (c), Supporting Information reveal the formation of etch pits of various diameters and depth profiles opened by 40 and $400 \mathrm{eV}$ ion impacts, respectively. In this study, $400 \mathrm{eV}$ energy level was used for the etching process.

SiGe Growth: Four effusion cells which are loaded with high purity intrinsic Si and Ge were heated up to $1350-1400^{\circ} \mathrm{C}$. The growth rates of each cell were optimized to obtain a growth rate of $0.60 \mathrm{~A}^{\circ} \mathrm{s}^{-1}$ for co-deposition by quartz crystal microbalance
(QCM). Si and Ge vapors were co-deposited at equal composition rates of $50 \%$ at $550^{\circ} \mathrm{C}$ substrate temperature. For homogenous coating, sample holder was rotated at $10 \mathrm{rpm}$. The MBE system was optimized before the deposition process so that the thickness of the deposition could be well controlled as also demonstrated in our previous study. ${ }^{[15]}$ In addition to the deposition thicknesses used in this study, various deposition thicknesses and different nanopit geometries could be tried, however, due to the volume restriction in our study this could not be possible.

Annealing: Samples were post-annealed at $750{ }^{\circ} \mathrm{C}$ for $30 \mathrm{~min}$ after the completion of the deposition process.

Characterizations: HR-SEM images were taken by Field Emission JEOL SEM 7001F at $15-20 \mathrm{kV}$ acceleration voltages in secondary electron imaging (SEI) mode where EDS measurements were acquired by Oxford instruments INCAx-act detector. Raman Spectroscopy measurements were held by Thermo Scientific DXR Raman Spectroscopy system with a $532 \mathrm{~nm}$ laser excitation at $3 \mathrm{~mW}$ laser output power. The AFM measurements were done using Parkafm SSS-NCHR sharp tip with $2 \mathrm{~nm}$ tip radius in non-contact mode by Park Systems XE-100E to ensure the accuracy of the measurement. As a final investigation, semiconducting analysis was performed with Keithley 4200-SCS Model FPP Semiconductor Analyzing System. Before etching, treated surface was properly checked by RHEED applying $10 \mathrm{keV}$ at a glancing angle around $1^{\circ}$ (Figure S3, Supporting Information).

Results and Discussion: Figure 1 shows the mean size, depth, and angle of the Si nanopits formed on the Si substrate upon $\mathrm{Ar}^{+}$ plasma etching by $400 \mathrm{eV}$. The schematic depicts the Ge NCs
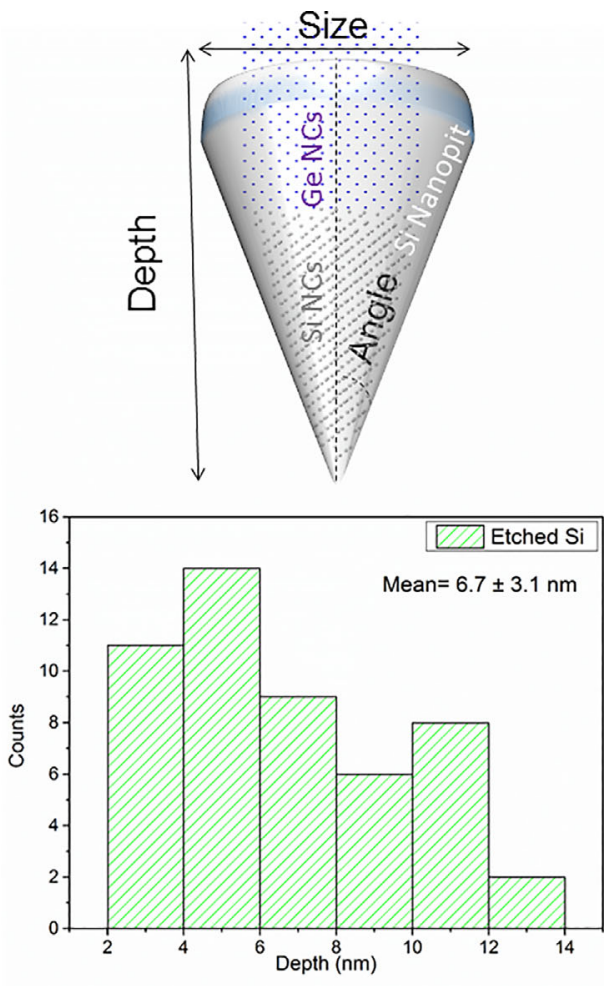
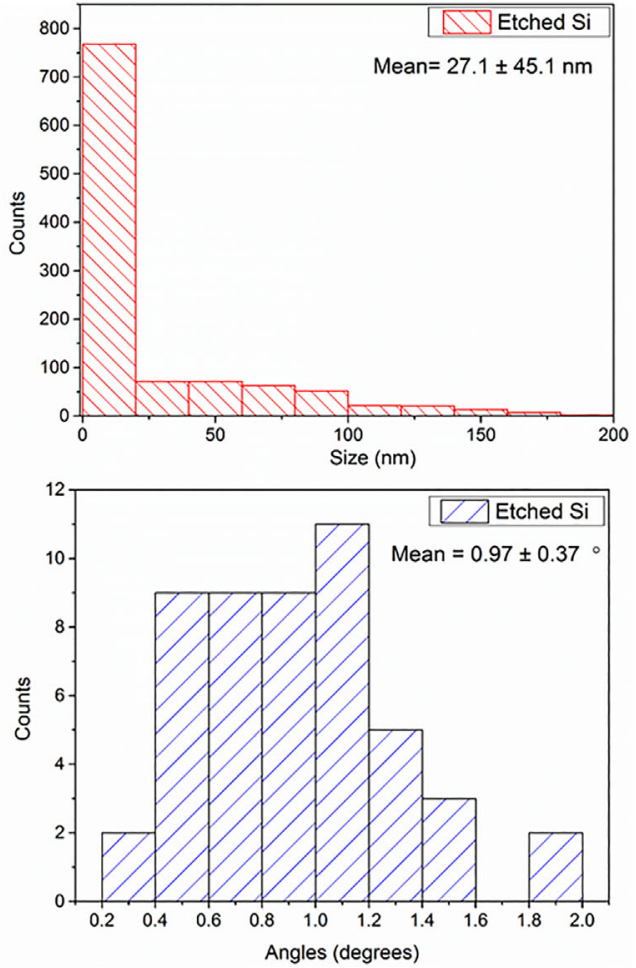

Figure 1. The schematic and the size distributions of the width, the depth, and the angles of the Si nanopits formed upon $\mathrm{Ar}^{+}$plasma etching. 


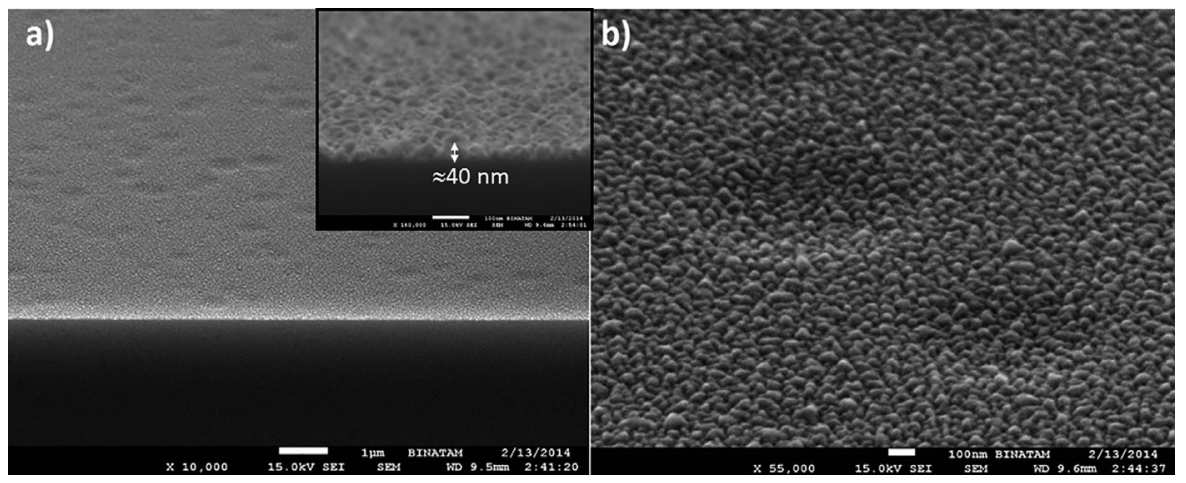

Figure 2. Inclined view SEM micrographs of Sample40 at low (a) and high (b) magnification imaging. The inset in (a) shows the cross-sectional view where the coating thickness is $\approx 40 \mathrm{~nm}$.

deposited on top of the Si NCs inside one Si nanopit. The mean size of the depth is $6.7 \mathrm{~nm} \pm 3.1 \mathrm{~nm}$. Thus, two special conditions for the deposition were chosen to grow the SiGe NCs. The first condition of the deposition thickness was chosen to be $4 \mathrm{~nm}$ smaller than the depth of the Si nanopits. The second condition was adjusted to be larger than the depth of the mean size of the Si nanopits as $40 \mathrm{~nm}$.

Figure 2 shows the inclined view SEM micrographs of Sample40 with a larger scale (Figure 2(a)) and the zoomed-in one (Figure 2(b)). Size-selective Ge islanding was not observed on the $40 \mathrm{~nm}$ SiGe grown sample (Sample40). Accordingly, the etch pits show no sign of the attraction center for Ge islanding due to fact that the size of the deposited film was much larger than the depth of pits. As seen in Figure 2(b), Ge nanoislands are distributed homogeneously on the etched template surface without forming any selective behavior.

AFM image of the sample before deposition is shown in the inset of Figure 3(b). Two distinctive patterns having five pits (above in the red rectangle) and four pits (below) were chosen to illustrate the appearance after the deposition process. After $4 \mathrm{~nm}$ growth and annealing processes of the SiGe layer (Sample4), it has been observed that Ge islanding phenomena is observed on the etched pit patterns (Figure 3(a)). The red and green line segments indicate the height profile of the Ge islands those were nucleated selectively on the etched pit points exactly. On the other hand, dark blue arrows show that the relatively smaller

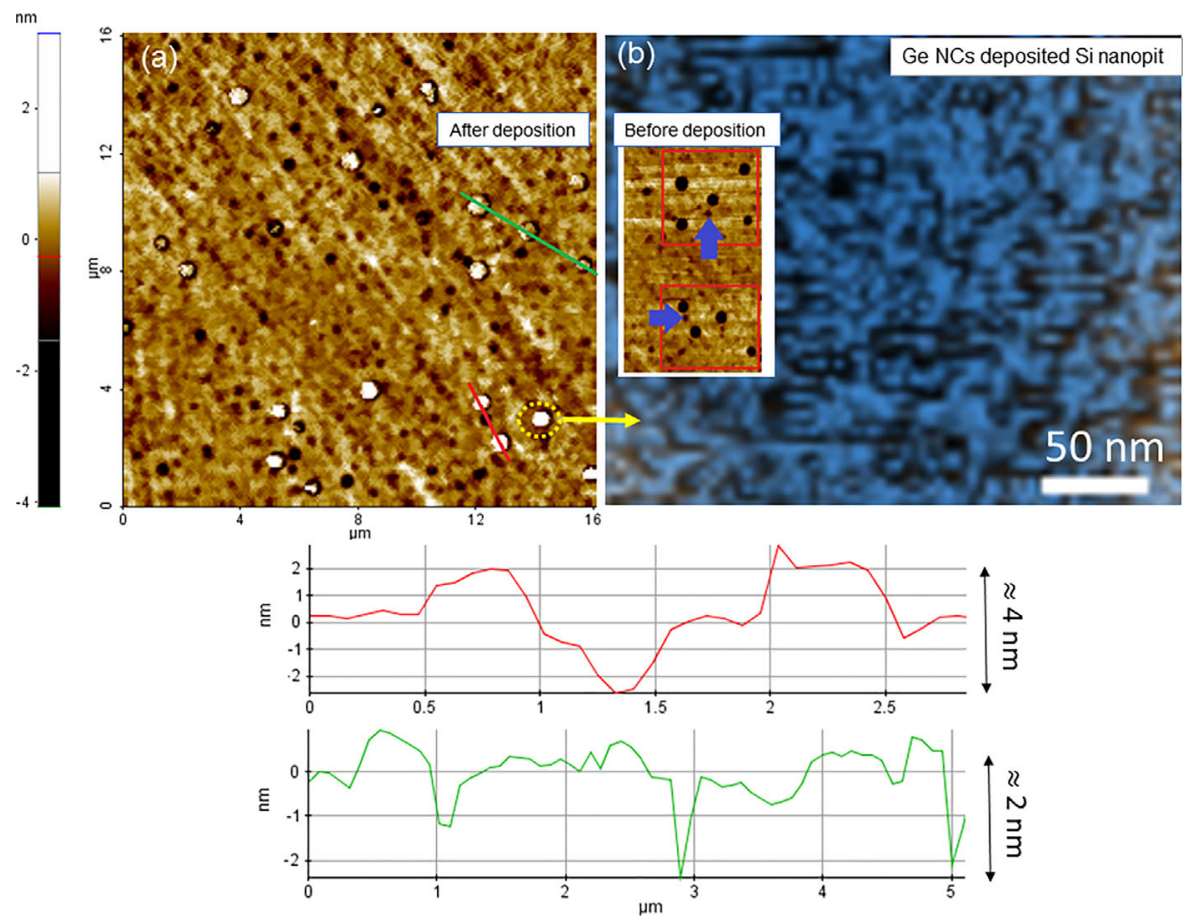

Figure 3. The AFM images and the line profiles of $4 \mathrm{~nm}$ thick SiGe grown sample (Sample4) before (the inset) and after deposition (left image). Height profiles of red and green line segments on the left image are given below. In the right image, Ge NCs filled inside the Si nanopit are shown using AFM technique from one nanopit (marked by yellow dashed circle). 
sized and shallow pits were not observed to promote nucleation of the Ge islands. This was considered to be due to the size of the nanopits. The mean size of the Si pits filled by the Ge NCs were $452 \pm 152 \mathrm{~nm}$. The unfilled Si nanopits have a mean size of $280 \pm 65 \mathrm{~nm}$. These two results are quite larger than the mean size the Ge NCs $(4.7 \pm 1.6 \mathrm{~nm})$. Therefore, it suggests that the pit size does not profoundly affect on the selectivity of the Ge NCs deposition. Nevertheless, the surface migration play role in very small depth (relatively more shallow pits) and yielding the unfilled Si nanopits (ca. $1.5 \mathrm{~nm}$ ) whereas Ge NCs selectively were nucleated only within those having larger depths (ca. $3 \mathrm{~nm}$ ).

The mean size out of 1337 nanoislands were found to be $\approx 11.4 \pm 6.9 \mathrm{~nm}$. The corresponding size distribution of the nanoislands is given in Figure 4(a). The co-existence of SiGe atoms in both samples were checked by the energy dispersive spectroscopy (EDS) measurement (Table 1 and Table S1, Supporting Information). As anticipated, Ge atomic percentage was increased in Sample40. Ge rate on the surface appears to be relatively small as energy dispersive X-rays penetrate much deep into Si substrate.

The dispersive Raman measurements were conducted from both of the samples. The broad peak for the Ge-Ge phonon vibration peak around $300 \mathrm{~cm}^{-1}$ was observed from Sample4. Firstly, the asymmetric peak in the first order Raman peak of Ge can be due to crystalline nature of Ge nanocrystals. ${ }^{[16]}$ On the other hand, not only finite size effect can cause broadening but also existence of disorder $\left(\left(v_{\mathrm{a}-\mathrm{Ge}}(\mathrm{LA}\right.\right.$ and $\mathrm{LO})=240 \mathrm{~cm}^{-1}$ and $v_{\mathrm{a}-\mathrm{Ge}}(\mathrm{TO})=275 \mathrm{~cm}^{-1}$ ) can lead to an increase in the width due to relaxation of momentum conservation rule. ${ }^{[17]}$ Furthermore, the strain between the mismatch of $\mathrm{Ge}$ and $\mathrm{Si}$ lattices can also cause a shift to the higher frequencies. ${ }^{[18]}$ This means that Gaussian distribution can implement, in terms of the analysis of the Raman data concerned, any contribution from disorders whereas the PCM can estimate the crystallite size. Therefore, one should consider these contributions together when interpreting the Raman data. This approach was previously applied by our group for $\mathrm{Ge}^{[19]}$ and by others for $\mathrm{Si}^{[20]}$ The size of the Sample4 based on the PCM was found to be $2 \mathrm{~nm}$ $\left(F W H M=63.6 \mathrm{~cm}^{-1}\right)$. Furthermore, there are signs of local disorder and strain remained even after the annealing process (red and black arrows shown, respectively, in Figure 4(c)). Si-Si and $\mathrm{Ge}-\mathrm{Ge}$ phonon vibration peaks of Sample40 are illustrated in Figure 4(d). Si-Si vibration peaks that arose from the substrate and the grown film are apparent at around $518 \mathrm{~cm}^{-1}$ whereas the
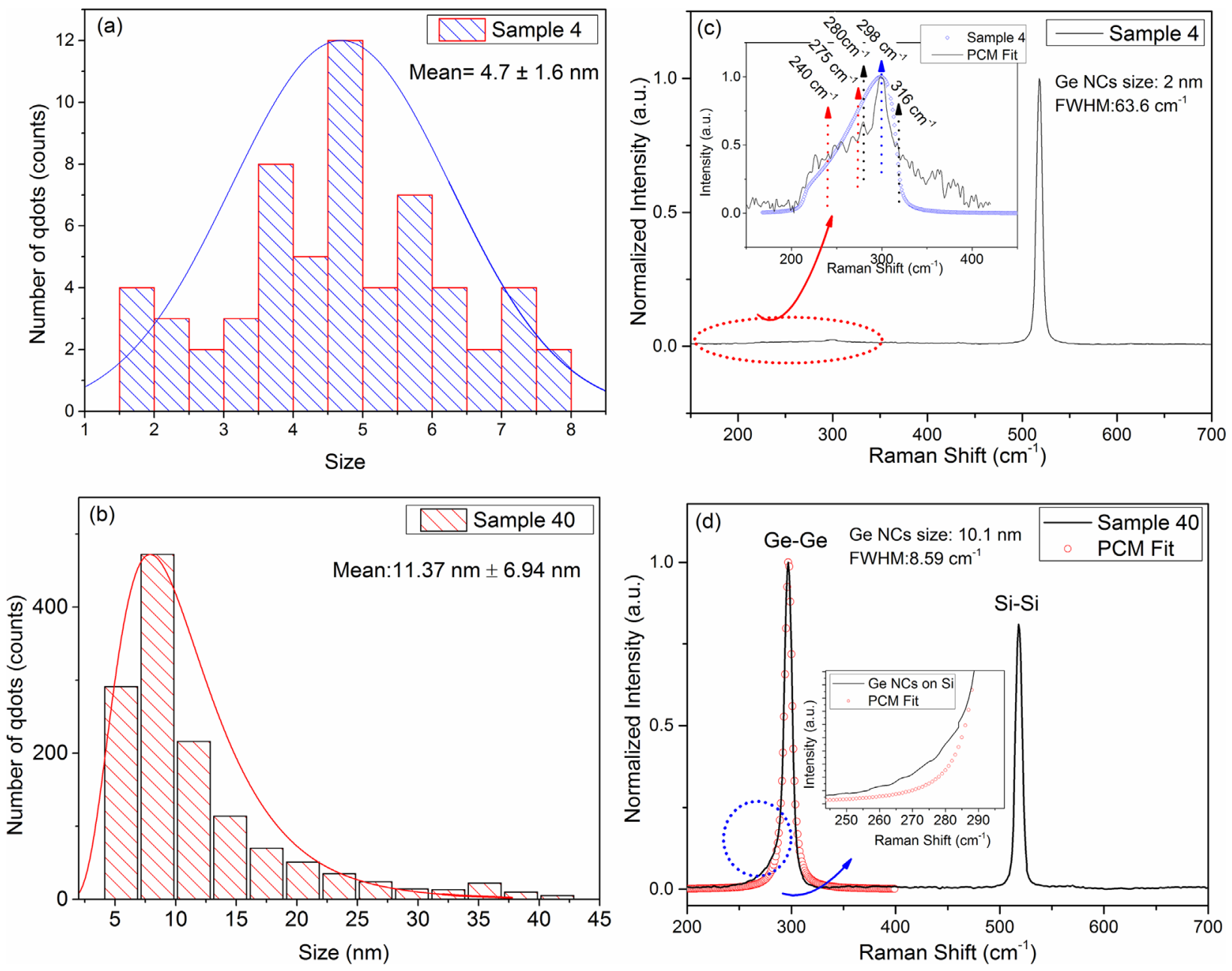

Figure 4. The size distributions and the dispersive Raman spectra of Sample4 and Sample40. 
Table 1. Elemental analysis using EDS measurement and Si-Ge atomic percentage values of Sample4 and Sample40.

\begin{tabular}{lcc}
\hline Atomic \% & Sample4 & Sample40 \\
\hline $\mathrm{Si}$ & 99.81 & 98.69 \\
$\mathrm{Ge}$ & 0.19 & 1.31 \\
\hline
\end{tabular}

strong Ge-Ge peak is observed at $291 \mathrm{~cm}^{-1}$ due to the Ge layer. These two peaks compared to their bulk crystalline counterparts are shifted to lower frequencies. This suggests both $\mathrm{Si}$ and $\mathrm{Ge}$ carries characteristics of nanocrystalline samples as suggested by phonon confinement model (PCM). ${ }^{[21,22]}$ In addition to the asymmetric shape and the shift of the phonon modes to lower frequencies, the relative intensity between $\mathrm{Si}$ and $\mathrm{Ge}$ layers $(I(\mathrm{Ge}) / I(\mathrm{Si}))$ can be used to indicate the difference between the Raman data of the different samples. ${ }^{[18]}$ Thus, the Raman data of Sample4 and Sample40 can also be distinguished using the relative intensity ratio in each Raman data between the $\mathrm{Ge}-\mathrm{Ge}$ phonon mode/the $\mathrm{Si}-\mathrm{Si}$ phonon mode. $I(\mathrm{Ge}) / I(\mathrm{Si})$ is $0.025 /$ $1=0.025$ for the Sample4 and $1 / 0.81=1.24$. This further supports the sizes found by the PCM and the SEM.

The crystallite size of Ge has been estimated to be $10.1 \mathrm{~nm}$ $\left(\mathrm{FWHM}=8.59 \mathrm{~cm}^{-1}\right.$ ) using the PCM. This seems to be mostly consistent with the results found from the SEM (Figure 3(a)) with small portion of disagreement due to possible amorphous contents. For the potential applications of the obtained samples, we have proceeded with the electrical investigations. The $I-V$ measurements from Sample40 yield a diode characteristics (Figure 5). Here, without the etching process applied prior to the deposition of SiGe nanoislands, an ohmic behavior was observed. This suggests that the etching process is required not only for the spatial selectivity of the deposition of the SiGe nanoislands but also for enhancing the diode characteristics. In Four Point Probe (FPP) analysis, lateral electrical data is taken by four conductive pins that are gently contacted on the surface of the films without damaging the crystal structure. Since the substrate has high resistivity compared to thin film segment, conduction path is assumed to be within the grown segment as it

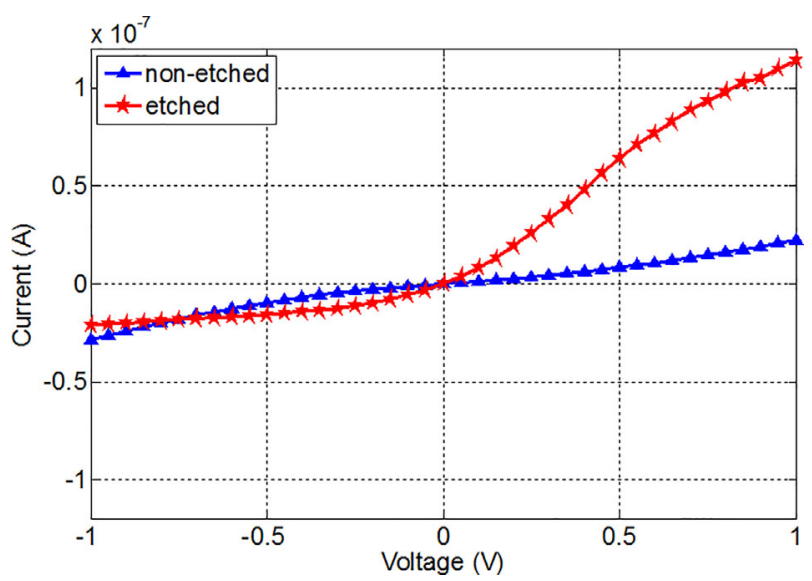

Figure 5. Lateral $I-V$ measurements of SiGe layers by FPP method for test sample (non-etched) and Sample40 (etched). has been verified by the bare substrate measurement values. It is also ensured that ohmic contact was formed between the pins and the surface without any metal-semiconductor effect. I-V curves of etched and non-etched SiGe films between $\pm 1 \mathrm{~V}$ are depicted in Figure 5 in red and dark blue, respectively. Accordingly, current values ranging below $\mu \mathrm{A}$ values indicate that rich crystalline Ge content enhances the current flow reducing the energy barrier between the valence band and the conduction band. It is possible that the slight discrepancy in the current values might be governed by the geometry of top layer as the etched surface atoms would have more distorted structure. This effect eventually leaded in quite less hole mobility than the non-etched test sample and resulted in diode type electrical behavior.

In summary, we showed the selective MBE deposition of SiGe nanoislands only within the Si nanopits whose selectivity was found to be correlated with the depth of the Si nanopits. When the deposition thickness was larger than the etched depth, the size selectivity were observed to be vanished. We found that about the deposition thickness of $4 \mathrm{~nm}$ close to the mean depth of the $\mathrm{Si}$ pits produced the selective growth of $\mathrm{Ge} \mathrm{NCs} / \mathrm{Si} \mathrm{NCs}$ inside the Si nanopits. We observed that such selective behavior was disappeared when relatively larger deposition thickness (e.g., $40 \mathrm{~nm}$ ) was used and the deposition was all along the surface of the Si substrate. Furthermore, the results suggest for the Sample4 that the surface migration yields the unfilled $\mathrm{Si}$ nanopits (ca. $1.5 \mathrm{~nm}$ ) while Ge NCs selectively were nucleated only within those having larger depths (ca. $3 \mathrm{~nm}$ ). The filled Ge $\mathrm{NCs}$ were observed to be grown in crystalline form with a very small portion of the amorphous contents.

\section{Supporting Information}

Supporting Information is available from the Wiley Online Library or from the author.

\section{Acknowledgments}

All authors contributed equally to this work. This work was supported by Fatih University Research Council under the project number of P500661201_B (2170). All the experimental studies were carried out in Bionanotechnology Research and Development Center (BINATAM).

\section{Conflict of Interest}

The authors declare no conflict of interest.

\section{Keywords}

dry etch, molecular beam epitaxy, selective growth, SiGe nanoislands, surface migration

Received: December 10, 2017

Revised: March 9, 2018

Published online: March 24, 2018 
[1] Q. Chen, Y. Song, K. Wang, L. Yue, P. Lu, Y. Li, Q. Gong, S. Wang, Nanoscale 2015, 7, 8725.

[2] J. Liu, X. Sun, R. Camacho-Aguilera, L. C. Kimerling, J. Michel, Opt. Lett. 2010, 35, 679.

[3] R. Koerner, M. Oehme, M. Gollhofer, M. Schmid, K. Kostecki, S. Bechler, D. Widmann, E. Kasper, J. Schulze, Opt. Express 2015, 23, 14815 .

[4] J. Michel, J. Liu, L. C. Kimerling, Nat. Photonics 2010, 4, 527.

[5] M. Karakiz, B. Toydemir, B. Unal, L. Colakerol Arslan, Eur. Phys. J. Appl. Phys. 2014, 65, 20403.

[6] K.-H. Shim, H. Deok Yang, Y.-H. Kil, J.-H. Yang, W.-K. Hong, J.-J. Kim, S. Kang, T. Soo Jeong, T. Sung Kim, Mater. Sci. Semicond. Process. 2013, 16, 126.

[7] A. F. Abd Rahim, M. R. Hashim, N. K. Ali, A. M. Hashim, M. Rusop, M. H. Abdullah, Microelectron. Eng. 2014, 126, 134.

[8] V. Poydenot, R. Dujardin, F. Fournel, J. L. Rouvière, A. Barski, J. Cryst. Growth 2005, 278, 83.

[9] Z. Zhong, H. Lichtenberger, G. Chen, M. Mühlberger, C. Schelling, J. Myslivecek, A. Halilovic, J. Stangl, G. Bauer, W. Jantsch, F. Schäffler, Microelectron. Eng. 2006, 83, 1730.

[10] C. Dais, H. H. Solak, E. Müller, D. Grützmacher, Appl. Phys. Lett. 2008, 92, 143102
[11] C. Dais, G. Mussler, T. Fromherz, E. Müller, H. H. Solak, D. Grützmacher, Nanotechnology 2015, 26, 255302.

[12] C. Dais, G. Mussler, H. Sigg, E. Müller, H. H. Solak, D. Grützmacher, J. Appl. Phys. 2009, 105, 122405.

[13] K. Peng, X. Wang, L. Li, X.-L. Wu, S. Lee, J. Am. Chem. Soc. 2010, $132,6872$.

[14] A. Birner, R. B. Wehrspohn, U. M. Gösele, K. Busch, Adv. Mater. 2001, 13, 377.

[15] i. Şeker, A. Karatutlu, O. Gürbüz, S. Yanık, Y. Bakış, M. Karakız, Appl. Phys. A 2018, 124, 47.

[16] C. E. Bottani, C. Mantini, P. Milani, M. Manfredini, A. Stella, P. Tognini, P. Cheyssac, R. Kofman, Appl. Phys. Lett. 1996, 69, 2409.

[17] Y. Sasaki, C. Horie, Phys. Rev. B 1993, 47, 3811.

[18] A. B. Talochkin, A. G. Cherkov, Nanotechnology 2009, 20, 345702.

[19] A. Karatutlu, M. Song, A. P. Wheeler, O. Ersoy, W. R. Little, Y. Zhang, P. Puech, F. S. Boi, Z. Luklinska, A. V. Sapelkin, RSC Adv. 2015, 5 , 20566.

[20] A. F. F. Beloto, P. G. Abramof, N. G. Ferreira, A. Y. Ueta, J. Non Cryst. Solids 2004, 340, 139.

[21] H. Richter, Z. P. Wang, L. Ley, Solid State Commun. 1981, 39, 625.

[22] I. H. Campbell, P. M. Fauchet, Solid State Commun. 1986, 58, 739. 